\title{
Study of the Standard Model Higgs boson decaying to taus at CMS
}

\author{
Valeria Botta*t \\ Deutsches Elektronen-Synchrotron (DE) \\ E-mail: valeria.botta@cern.ch
}

The most recent search for the Standard Model Higgs boson decaying to a pair of $\tau$ leptons is performed using proton-proton collision events at a centre-of-mass energy of $13 \mathrm{TeV}$, recorded by the CMS experiment at the LHC. The full 2016 dataset, corresponding to an integrated luminosity of $35.9 \mathrm{fb}^{-1}$, has been analysed. The Higgs boson signal in the $\tau^{+} \tau^{-}$decay mode is observed with a significance of 4.9 standard deviations, to be compared to an expected significance of 4.7 standard deviations. This measurement is the first observation of the Higgs boson decay into fermions by a single experiment.

The European Physical Society Conference on High Energy Physics 5-12 July 2017

Venice, Italy

* Speaker.

${ }^{\dagger}$ On behalf of the CMS Collaboration. 


\section{Introduction}

In July 2012 the ATLAS and CMS Collaborations announced the discovery of a new particle $[1,2,3]$, compatible with the neutral scalar boson predicted by the Brout-Englert-Higgs mechanism of electroweak symmetry breaking $[4,5,6]$, commonly known as the Higgs boson (H). From a combination of ATLAS and CMS measurements [7], the mass of the Higgs boson has been determined to be $125.09 \pm 0.21$ (stat.) \pm 0.11 (syst.) $\mathrm{GeV}$. To establish the mass generation mechanism for fermions, it is necessary to probe the direct coupling of the Higgs boson to such particles. The most sensitive decay channel is $\mathrm{H} \rightarrow \tau^{+} \tau^{-}$due to the expected large event rate in the Standard Model (SM) and small contribution from background events with respect to other fermionic decay modes. Searches for the Higgs boson decaying to a tau pair at the LHC were performed by ATLAS and CMS using pp collision data at $\sqrt{s}=7$ and $8 \mathrm{TeV}$, the combination of the results from both experiments [8] resulted in an observed (expected) significance of 5.5 (5.0) $\sigma$. In the following, the search for the Higgs boson decaying to a tau pair using pp collision data at $\sqrt{s}=13 \mathrm{TeV}$ recorded by the CMS experiment [9] is presented, summarising the analysis strategy and the main results. The full 2016 dataset, corresponding to an integrated luminosity of $35.9 \mathrm{fb}^{-1}$, has been analysed [11].

\section{Analysis strategy}

In order to select $\mathrm{H} \rightarrow \tau^{+} \tau^{-}$events, several final states need to be taken into account, because tau leptons decay inside the CMS detector, either leptonically to an electron $(e)$ or a muon $(\mu)$ and neutrinos $(\mathrm{BR}=35 \%)$, or hadronically $\left(\tau_{h}\right)$ to pion(s) and neutrino $(\mathrm{BR}=65 \%)$. The reconstruction of hadronic tau decays [10] is based on the number of tracks and strip-shaped electromagnetic energy deposits, and a MVA discriminator is used to reject fakes from jets. For this analysis, the chosen working point corresponds to a tau identification efficiency of $60 \%$ for a jet misidentification rate of $1 \%$. The four most likely final states for $\tau \tau$ pairs, namely $\tau_{h} \tau_{h}, \mu \tau_{h}, e \tau_{h}$ and $e \mu$, are considered in the analysis. Events are selected in data using a combination of single lepton, dilepton and di-tau triggers, according to the different final states, and they are further categorised into the following mutually exclusive categories:

1. the 0 -jet category, targeting gluon-fusion Higgs production, contains events with no jets with $p_{T}>30 \mathrm{GeV}$ and $|\eta|<4.7$;

2. the VBF category, targeting vector-boson fusion production, contains events with two jets with invariant mass $\mathrm{m}(\mathrm{jj})>300 \mathrm{GeV}$;

3. the boosted category, that includes all other events not entering one of the previous categories, to account for gluon fusion production accompanied by jets, softer VBF events and VH production with the vector boson decaying hadronically.

The signal is extracted with a maximum likelihood fit to two-dimensional distributions of two sensitive variables. One variable is always the mass of the di-tau system, while the second variable has been chosen to disentangle the signal from the most important background processes in that channel and category. A total of twelve two-dimensional distributions is included in the final fit, one per channel and per category. 
The main irreducible background in all channels is represented by $\mathrm{Z} \rightarrow \tau^{+} \tau^{-}$events, it is estimated from Monte Carlo simulation and several corrections derived from data are applied. In the $\tau_{h} \tau_{h}$ channel the main reducible background is QCD multi-jet production, that is estimated with a data-driven method using events with less isolated tau leptons. In the other channels the QCD background is estimated from data with same-sign tau leptons. The $\mathrm{W}+\mathrm{jets}$ background is particularly relevant for the $\mu \tau_{h}$ and $e \tau_{h}$ channels and it is suppressed by requiring the invariant mass of the lepton+transverse missing energy system being less than $50 \mathrm{GeV}$. In the $e \mu$ channel, $t \bar{t}$ events constitute an important background, that can be rejected exploiting the fact that in tau decays the directions of neutrinos and leptons tend to be closer than in $t \bar{t}$ decays. The normalisations of the QCD, $\mathrm{W}+\mathrm{jets}$ and $t \bar{t}$ backgrounds are derived from data thanks to dedicated control regions included in the final fit. Other minor backgrounds like single top and diboson production are estimated from simulation.
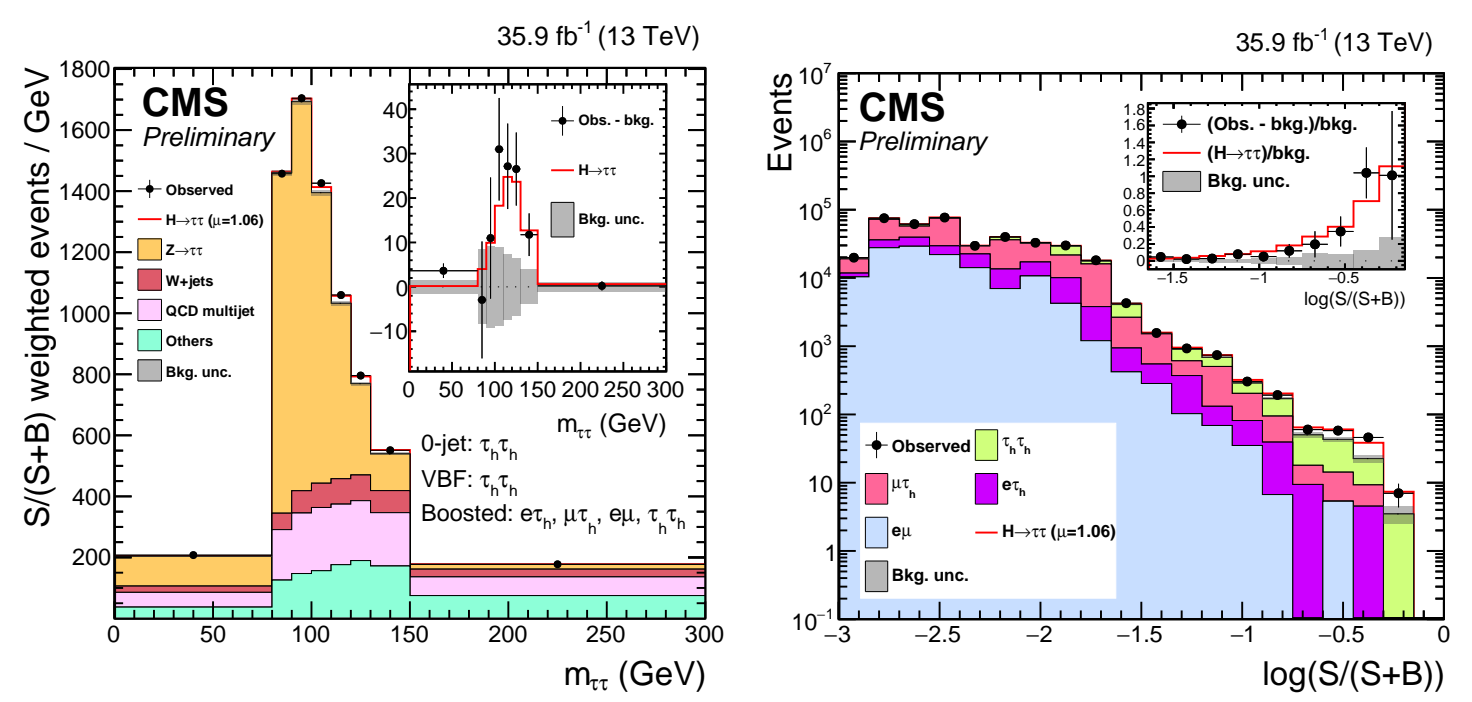

Figure 1: Left: Combined observed and predicted di-tau mass distributions for all categories of the $\tau_{h} \tau_{h}$ channel and the boosted categories of the other channels. The normalisation of the predicted background distributions corresponds to the result of the global fit, while the signal is normalised to its best fit signal strength. The events are weighted according to the expected to $S /(S+B)$. Right: Distribution of the decimal logarithm of the ratio between the expected signal and the sum of expected signal and expected background in each bin of the mass distributions used to extract the results, in all signal regions. More details can be found in [11].

\section{Results}

As a result of the global fit to the 2D distributions and the control regions, an excess of events in data compatible with the expected SM Higgs signal is observed. The left panel of Figure 1 shows the weighted expected and observed di-tau mass distribution combined for the most sensitive categories and channels, while the right panel shows the distribution of the decimal logarithm of the ratio between the expected signal and the sum of expected signal and expected background in each bin of the mass distributions used to extract the results, in all signal regions. The inset plots 
show the data after subtracting the expected background, compared to the $\mathrm{H} \rightarrow \tau^{+} \tau^{-}$signal with the fitted signal strength, clearly showing an excess of events in data that is well described by the SM Higgs signal. The excess is quantified by calculating the corresponding local $\mathrm{p}$-value using a profile likelihood ratio test statistic, and amounts to $4.9 \sigma$, the expectation for the SM Higgs boson with $m_{H}=125.09 \mathrm{GeV}$ being $4.7 \sigma$. The observed number of signal events is the one predicted for the SM Higgs boson, multiplied by a signal strength modifier $\mu$ treated as a free parameter in the fit. The best fit value for the signal strength is $\mu=1.06_{-0.24}^{+0.25}$ and is compatible among final states and with the SM expectation within the uncertainties. The main contribution to the signal strength uncertainty is statistical. Among the experimental uncertainties that affect the shapes of the di-tau mass, the most important are the ones related to the hadronic tau reconstruction, namely the tau energy scale uncertainties for genuine (1.2\%) and lepton-fake taus (1.5-3\%) and the scale uncertainties on the missing transverse energy, that are calculated event by event. This measurement is the first observation of the Higgs boson decay to fermions in a single experiment. The final result, also including a combination with the 7 and $8 \mathrm{TeV} \mathrm{H} \rightarrow \tau^{+} \tau^{-}$analysis from CMS became available after this Conference and can be found in [12].

\section{References}

[1] ATLAS Collaboration, Observation of a new particle in the search for the Standard Model Higgs boson with the ATLAS detector at the LHC, Phys. Lett. B 716 (2012) 1, doi:10.1016/j.physletb.2012.08.020"

[2] CMS Collaboration, Observation of a new boson at a mass of $125 \mathrm{GeV}$ with the CMS experiment at the LHC, Phys. Lett. B 716 (2012) 30, doi:10.1016/j.physletb.2012.08.021

[3] CMS Collaboration, Observation of a new boson with mass near $125 \mathrm{GeV}$ in pp collisions at $\sqrt{s}=7$ and 8 TeV, JHEP 06 (2013) 081, doi:10.1007/JHEP06(2013)081

[4] F. Englert and R. Brout, Broken symmetry and the mass of gauge vector mesons, Phys. Rev. Lett. 13 (1964) 321, doi:10.1103/PhysRevLett.13.321

[5] P. W. Higgs, Broken symmetries, massless particles and gauge fields, Phys. Lett. 12 (1964) 132, doi:10.1016/0031-9163(64)91136-9

[6] P. W. Higgs, Broken symmetries and the masses of gauge bosons, Phys. Rev. Lett. 13 (1964) 508, doi:10.1103/PhysRevLett.13.508

[7] ATLAS and CMS Collaborations, Combined Measurement of the Higgs Boson Mass in pp Collisions at $\sqrt{s}=7$ and 8 TeV with the ATLAS and CMS Experiments, Phys. Rev. Lett. 114 (2015) 191803, doi:10.1103/PhysRevLett.114.191803

[8] ATLAS and CMS Collaborations, Measurements of the Higgs boson production and decay rates and constraints on its couplings from a combined ATLAS and CMS analysis of the LHC pp collision data at $\sqrt{s}=7$ and $8 \mathrm{TeV}$, JHEP 08 (2016) 045, doi:10.1007/JHEP08(2016)045

[9] CMS Collaboration, The CMS Experiment at the CERN LHC, 2008 JINST 3 S08004, doi:10.1088/1748-0221/3/08/S08004

[10] CMS Collaboration, Performance of reconstruction and identification of $\tau$ leptons in their decays to hadrons and $v_{\tau}$ in LHC Run-2, CMS-PAS-TAU-16-002, https://cds.cern.ch/record/2196972 
[11] CMS Collaboration, Observation of the SM scalar boson decaying to a pair of $\tau$ leptons with the CMS experiment at the LHC, CMS-PAS-HIG-16-043, http://cds.cern.ch/record/2264522

[12] CMS Collaboration, Observation of the Higgs boson decay to a pair of $\tau$ leptons, Submitted to Phys. Lett. B (2017), http://cds.cern.ch/record/2276465 